\title{
A MODEL FOR R AQR FROM HST
}

\author{
Denis BURGARELLA ${ }^{1}$, Manfred VOGEL ${ }^{2}$ and Francesco PARESCE ${ }^{13}$ \\ 1 STScI, 3700 San Martin Drive, Baltimore, Md 21218 \\ 2 Institute of Astronomy, ETH Zürich, Switzerland \\ 3 Affiliated to the Astrop. Division, Space Science Department, ESA
}

\begin{abstract}
High spatial resolution observations $\mathrm{R}$ Aqr have been carried out with the HST/FOC. R Aqr is the nearest symbiotic star, i.e. an interacting system consisting of a cool giant star and a hot ionizing source embedded in a larger and complex nebula. We suggest, here, that the binary system is composed of a Mira and a hot stellar source with a radiation temperature of $T^{*} \approx 40000 \mathrm{~K}$, a luminosity of $L \approx 10 \mathrm{~L}_{\odot}$ and a radius $\mathrm{R} \lesssim 0.1 \mathrm{R}_{\odot}$. Mass transfer between the two components of the system occurs via capture of the Mira wind. Of the plausible mechanisms that may produce the $\mathrm{R}$ Aqr jet, an accretion/ejection scenario or the collision of winds seem to be unlikely, but spectral observations of the core are needed to conclude. Another possible origin is based on a spherically symmetric stellar wind that sweeps out a cavity in an ambient medium. This bubble elongates in the directions of least resistance, and matter eventually flows out through two symmetric nozzles. Comparison with models shows that the emission in the NE and SW outer features of the jets is due to a radiative shock but the pre-shock gas must be partially photoionized by the central hot stellar source.
\end{abstract}

\section{Introduction}

R Aquarii is the nearest symbiotic star (250 pc, Whitelock 1987), i.e. an interacting binary consisting of a cool Mira and a hot ionizing source. The HST images (Paresce et al. 1991) confirmed that it is also the nearest astrophysical jet. We have therefore a unique opportunity to study a smaller scale reproduction of the extragalactic jets. An accretion disk, axi-symmetric collimated outflow, curvature of the stream, non-thermal radio emission, collision with a dense external medium producing a decollimation of the jet are all mechanisms that might be occuring in $R$ Aquarii.

\section{HSR Observations}

Fig. 1 presents the F120M inner nebula as observed by the FOC. The structure of the two radio features $\mathrm{C}_{1}$ and $\mathrm{C}_{2}$ can be superposed very well on the HST images. The collimated jet of $\mathrm{R}$ Aqr clearly appears in the F501N image (Paresce et al. 1991). The morphology of the feature $\mathrm{C}_{2}$ is remarkably similar to a Herbig-Haro object, as would produce a collimated wind from the binary system and $\mathrm{C}_{1}$ should contain both stars. The radio feature $A$ has been divided in a filamentary area $A_{1}$ and a darker region $A_{2}$ (see Paresce et al. 1991). The counter-jet $A^{\prime}$ is also clearly visible. 


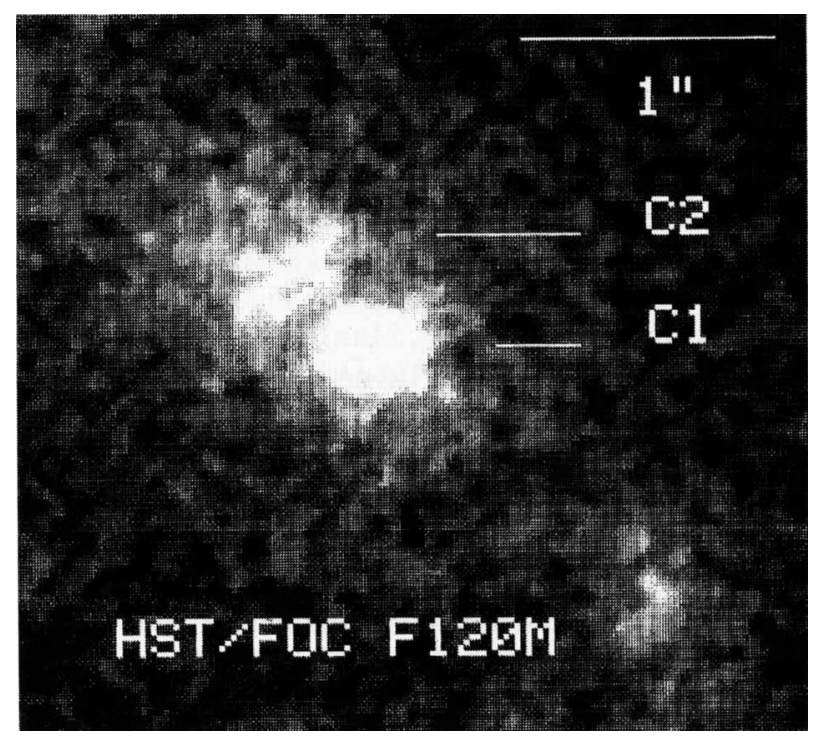

Fig. 1 - HST images of the inner $7.7 \times 7.7 \operatorname{arcsec}^{2}$ of R Aqr observed with the FOC through the F120M filter. The symbols are defined in the text. North is up and east to the left.

c)

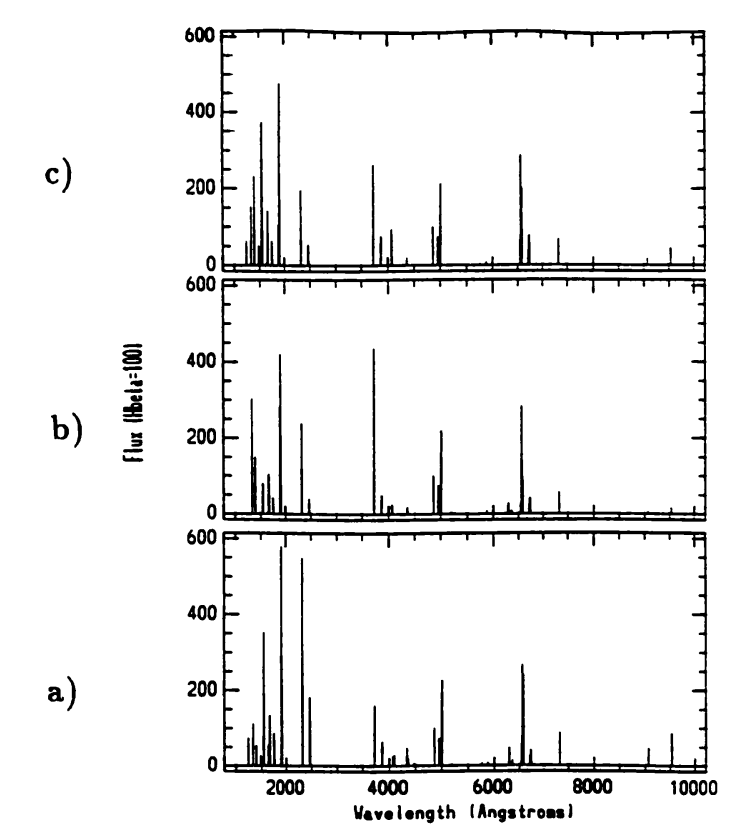

b)

Fig. 2 - Schematic representation of a) the observed spectrum of the NE jet, b) the best shock model for $v=100 \mathrm{~km} \mathrm{~s}^{-1}$ and c) our shock plus photoionization model $M_{1}$. The lines are represented as peaks with intensities relative to $H \beta=100$. 


\section{Comparison with models}

\subsection{Shock models}

Trying to clarify the physics governing the R Aqr system (Burgarella \& Paresce 1991), we recompare the radiative planar shock models of Hartigan, Raymond and Hartmann (1987), assuming equilibrium preionization, with the observed ultraviolet and optical fluxes for the NE jet (Hollis et al. 1991). The shock models shown here are for shock velocities between 90 and $110 \mathrm{~km} \mathrm{~s}^{-1}$ and "cosmic" abundances. Almost all observed line ratios in the NE jet are compatible with an "intermediate" model, which predicts ratios between the high state of the models with $v \gtrsim 100$ $\mathrm{km} \mathrm{s}^{-1}$ and the low state of the models with $v \lessgtr 100 \mathrm{~km} \mathrm{~s}^{-1}$.

\subsection{Photoionization}

The grid of calculated models spans a range in radiation temperature of $20000 \mathrm{~K}$ $\lesssim T^{*} \lesssim 70000 \mathrm{~K}$, and electron density of $10^{2} \mathrm{~cm}^{-3} \lesssim N_{e} \lesssim 10^{7} \mathrm{~cm}^{-3}$ and a variety of density distributions. None of these models can account for the observed line ratios. In particular, pure photoionization from the central source is not able to produce simultaneously the presence of observable He II $\lambda 1640$ and N V $\lambda 1240$ fluxes in the NE jet and no such emission in the compact central H II region.

\subsection{Shock + photoionization}

We propose a compound model for the NE jet of R Aqr where a central photon source preionizes the gas of a radiative shock. The shock region is simulated by a temperature structure. In the low temperature region, $T_{e}$ is calcuted by the equilibrium condition between radiative heating and cooling. The electron density rises from a low value in the high temperature region to high values in the low temperature region. The inner edge of the shock is at a distance of $2.3 \cdot 10^{16} \mathrm{~cm}$, which corresponds approximately to $6^{\prime \prime}$ from the central source. The extend of the emitting region is $7 \cdot 10^{15} \mathrm{~cm}$, corresponding to $\sim 2^{\prime \prime}$. Assuming that feature $\mathrm{B}$ is a spherical bubble, the total luminosity of the central star in R Aqr is estimated to be $L \approx 8 \mathrm{~L}_{\odot}$. The radiation temperature of the central star in $\mathrm{R}$ Aqr must be $T^{*} \lesssim 50000 \mathrm{~K}$, otherwise we expect an observable He II $\lambda 1640$ flux from the central $\mathrm{H}$ II region.

In Fig. 2, we compare the calculated spectrum of our model with the observations and the best radiative planar shock model of Hartigan, Raymond and Hartmann (1987). The code calculating the nebular emission is summarized in Nussbaumer and Vogel (1987) and detailed in Vogel (1990). Our final model (M1) consists of a central star with $T^{*}=40000 \mathrm{~K}, R^{*}=4 \cdot 10^{9} \mathrm{~cm}$, and the emission region as discussed above. A standard chemical gas composition was adopted. Although some discrepancies remain, we consider our model as, so far, the best qualitative description for the $\mathrm{NE}$ jet of R Aqr.

\section{The origin of the jet}

\subsection{Nozzle mechanism}

The nozzle mechanism (Raga and Cantó 1989 and references therein) is based on the interaction of a hot isotropic stellar wind with a cooler cloud of gas. The hot wind creates a cavity of hot gas which escapes only through the direction of 
least resistance along the axis of rotation or the lines of magnetic field. Due to instabilities, the external boundaries of the hubble could evolve in nozzles leading to the formation of jets. Then, these jets may be further collimated by interaction with the external medium. The external cool gas may have an origin in the capture of the Mira wind (Kenyon 1985), but a major problem in this configuration is the origin of the hot wind. This solution seems to be possible if the companion is a high mass loss rate subdwarf (or hot white dwarf).

\subsection{Collision of winds}

Solf and Ulrich (1985) have already shown that a wind is blowing out from the core of $\mathrm{R} \mathrm{Aqr}$ with polar velocities of $\sim 200 \mathrm{~km} \mathrm{~s}^{-1}$ exceeding the equatorial ones by a factor of 6-8. Moreover, in this scenario the X-ray emission source should be between the two stars. This seems therefore inconsistent with the collision of two isotropic winds, but we cannot definitely rule out this scenario and a quantitative hydrodynamic formulation (which does not exist at the moment) of such a wind collision is needed to compare with the data.

\subsection{Accretion disk}

Livio's (1988) condition shows that a quasi-steady accretion disk can form from capture of a stellar wind in R Aqr. In this scenario, we expect an accretion disk to be responsible for the jet outflow and also for the central photon source needed to explain the observed nebular emission. From the previous section, we find that the accretion disk or the boundary layer must be a photon source with a radiation temperature $\lesssim 50000 \mathrm{~K}$ and a luminosity $\approx 10 \mathrm{~L}_{\odot}$. But a disk temperature of about $30000 \mathrm{~K}$ would be accompained by a much higher boundary layer temperature, and the luminosity released in this boundary layer would be similar to the disk luminosity. Thus, an accretion disk seems to be inconsistent with the data and is therefore most likely not present in $\mathrm{R} \mathrm{Aqr}$ unless non-radiative processes play a major role.

This work will be detailed in a forthcoming paper in Astronomy \& Astrophysics. Acknowledgments: D. Burgarella thanks the ESA for a post-doctoral fellowship.

\section{References}

Burgarella, D. and Paresce, F. 1991, Ap. J., 370,590

Hartigan, P., Raymond, J. and Hartmann, L. 1987, Ap. J., 316,323

Hollis, J.M., Oliversen, R.J., Kafatos, M., Michalitsianos, A.G. and Wagner, R. M. and $1991, A p . J$. (in press)

Kenyon, S.J. 1985, "Interacting Binaries" in Eggleton, P.P. and Pringle, J.E. (eds.), Reidel Pub. Comp., Dordrecht, p. 179

Livio, M. 1988, "The Symbiotic Phenomenon", in Mikolajewska, J., Friedjung, M.,

Kenyon, S. C. and Viotti, R. (eds.), Kluwer Academic Pub., Dordrecht, p. 249

Nussbaumer, H. and Vogel, M. 1987, Astr. Ap., 213,137

Paresce, F. et al. 1991, Ap. J., (in press)

Raga and Cantó 1989 Ap. J., 344,404

Solf, J. and Ulrich, H. 1985, Ap. J., 148,274

Vogel, M. : 1990, Ph.D. Thesis, ETH Zürich, Switzerland

Whitelock, P. A. 1987, Pub. A.S.P., 99,573 